UDC 821.124

\title{
DE REMMII PALAEMONIS INDOLE VITIISQUE
}

\author{
Michael M. Pozdnev
}

St. Petersburg State University,

7-9, Universitetskaya nab., St. Petersburg, 199034, Russian Federation; m.pozdnev@spbu.ru

Univ. Trier, FB II Klassische Philologie Universitätsring

15, 54286, Trier, Deutschland; pozdnev@uni-trier.de

The disparaging report of Remmius Palaemon in Suet. DGR 23 contains his own seemingly absurd vainglorious dicta and a contemporary vis-à-vis joke aimed at his sexual appetites. The first cannot be as pointless as they seem given the great authority of Palaemon as a scholar and the second is textually unsatisfying, and thus not quite clear. The present study tries to improve on both, uncovering the actual witticism of Palaemon's words in the first case as well as the real meaning of his opponent's pun in the second. Thus, it turns out that behind the repulsive portrait drawn by Suetonius hides a much more sympathetic and less depraved personality.

Q. Remmium Palaemonem Persii magistrum (Vita, v. 11 Clausen) Quintiliani praeceptorem (Schol. ad Iuv. 4, 452; 7, 215), 'Artis grammaticae' auctorem ${ }^{1}$ et grammaticorum sui temporis principem (Suet. De gramm. et rhet. 23, 2; Plin. NH 14, 49) scriptoribus, qui vitam moresque eius posteritati mandare voluerunt, ipsum reprehensionis ansam dedisse satis constat. Nimia enim opinione ingenii sui fuit sapientemque se iactavit et hoc quidem coram discipulis. Inde ambitionis fama in schola, cuius rector fuit, orta scholasticorum more nutrita et usque ad imperatorum aures prolata est (Suet. loc. cit.). Mentionem vanitatis, quae nota mire in illo fuit, Plinius $(\mathrm{NH} \mathrm{14,50)}$ non ita longe post exitum Remmii fecisse videtur ${ }^{2}$ eandemque vanitatem testantia verba Suetonius inter alios rumores servavit, cum scripsit arrogantiam viri tantam fuisse,

ut M. Varronem porcum appellaret, secum et natas et morituras litteras iactaret, nomen suum in Bucolicis non temere positum sed praesagante Vergilio fore quandoque omnium poetarum ac poematum Palaemonem iudicem. gloriabatur etiam latrones quondam sibi propter nominis celebritatem parsisse (Gramm. 23, 4). ${ }^{3}$

Haec dicta quasi nuda et e sermonis contextu exempta accepimus causasque talia pronuntiandi ipse Suetonius ignorare videtur. Quam absurdum, qui grammaticus peritis-

${ }^{1}$ Fragm. ap. Charisium: GL V 534 sqq.; GRF II 75 sqq. De Palaemonis Arte grammatica: Barwick 1922; Collart 1938. „The first exclusively scholastic treatise on Latin Grammar“: Sandys 1903, 189. Aliter, sed ratione dubia: Kaster 2003, 230.

2 Ita etiam Kaster 2003, 229.

3 Suetonii textus hic et infra secundum Brugnoli 1972, 26.

(C) St. Petersburg State University, 2016 
simus putaretur, eum se litterarum non modo vindicem, sed etiam auctorem profiteri! Verumtamen litteras cum Palaemone et natas et morituras ad tres illas, quas Ti. Claudius invenit atque in separato volumine tractavit (Suet. Cl. 41, 3), ${ }^{4}$ pertinere posse suspicimus. Dixit fortasse Remmius, qui in ludo suo litteras Claudianas docere iuberetur, imperatorem de earum necessitate secum egisse atque approbationem suam obtinuisse. Quas tamen litteras supervacaneas non diu victuras, auctoritate sua, non voluntate principis in usu permansuras esse praedicavit. Superbe quidem dictum, verum etiam facete. Per verborum enim lusum litterarum scientiam eximiam ac singularem sibi arrogavit. Attamen devulgata Remmii sententia cum ambiguitate etiam salem suum perdidit.

Et Bucolicorum quoque auctorem, quem Musa inversionum amica posteris fatidicum reddidit, ${ }^{5}$ haud illepide adventum suum $E c l .3$, 50 vaticinari declaravit. Utrum serio haec affirmaverit an potius lascive, in sensus quos dicunt allegoricos iocose invectus, quis est qui dicat? ${ }^{6}$ Item incerti sumus, cur Varronem appelaverit porcum, sed quodam modo ad etymologias, quibus libri Varroniani exuberant, exprobrationem Remmii pertinere suspicamur. Ita enim porcos amat Varro, ut etiam Albam Longam ab sue alba ducat (De ling. Lat. 5, 144; de ipsius vocis porcus origine nonnihil hariolatur ibid. 97-98). Fortasse per iocum Remmius discipulis dixit in Varronem, quem Pythagoreorum doctrinam sectari credidit (cf. Plin. $N H 35,160),{ }^{7}$ porci animam transisse. Utcumque erat, non sine sale superbire Q. Remmium conicimus.

Quam quidem superbiam quaesitam meritis esse certe scimus. Nam adeo philologus erat, ut nomen eius diutius grammaticum significaret (v. Iuv. 7, 215-219; etiam 6, 452 Palaemonis artem grammaticam vocat). Illa de eximia doctrina gloria latrones adduxit, ut magistro parcerent. ${ }^{8} \mathrm{Ni}$ mirum arrogans fuit, cuius arrogantiae nec non auctoritatis gratia fieri potuit, ut Senecae philosophi (Plin. $N H$ 14, 51), cui philologorum grammaticorumve labor omnino contemptui erat (Ep. 108, 30 sqq.), et M. Antonii Liberalis (Suet. De gramm. frgm. cap. 38) odium moveret. ${ }^{9}$ Sui nimium cultor immodico balneorum usu luxuriae reprehensionem apud Suetonium meruit $(23,5)$. Vix tamen credere possumus pecuniam, quae e schola officinisque textrinis ${ }^{10}$ aliaque re familiari diligentissime aucta haud immodica Remmio conficeretur, sumptibus eius parum suffecisse (ibid.). Si ita fuisset, nullo modo in agro Nomentano rus mercari potuisset. Quo de fundo Stheneli vinitoris auxilio miram vindemiam faciebat: Plinius has uvas et Suetonius una voce laudant. Post decem autem curae annos quadruplicato pretio Senecae inimico suo ac contemptori vendidit (Plin. NH 14, 49-52).

Illud certe vanitatis vitium apud vv. dd. haud inusitatum Romanis autem omnium invisissimum (notum est illud Ciceronis nec vero est quicquam turpius vanitate: Off. 1, 150) primoribusque populi in homine liberto praecipue irridendum, causa fuit Tiberio et postea

${ }^{4}$ De quarum imaginibus atque originibus: Oliver 1949.

${ }^{5}$ Quocirca infantem illum, cui Ecl. 4 dedicavit Augusti aevo natum ideoque felicem aevi aurei praenuntium etiamnunc false interpretantur, sicut iam pridem interpretati sunt. Asinius Gallus olim, sibi $E c l$. 4 dedicatam esse proclamavit (Ascon., SD ad v. 11).

${ }^{6}$ L. Annaeus Cornutus, qui Persii magister alter post Remmium fuit, in commentariis, quorum fragmenta apud Servium servata sunt, Vergilium de vaniloquentia accusabat (GRF 29, 35). Eiusdem generis reprehensiones apud Persium invenies (1, 96-97).

${ }^{7}$ De Varrone etymologo Pythagoreo uberius: Del Bello 2007, 80-82; Kahn 2001, 88-90; Rawson 1985, 161.

${ }^{8}$ Kaster 2003, 238: "Since such toughs would typically be as uneducated as they were fierce, their clement respect for a man of culture would be doubly remarkable".

9 Ibid. 232: "P. was no doubt a provocative figure, whose success... aroused invidia in others."

10 Textrinum Palaemon statu adhuc servili, prius etiam quam litteras, didicit: Gramm. 23, 1. De vita eius copiosius: Kolendo 1985, 177-87. 
Claudio, cur dicerent nemini minus institutionem puerorum vel iuvenum conmittendam (Suet. De gramm. 23, 2). Minime tamen eis credendum est, qui propter hanc sententiam paediconem notissimum Remmium fuisse putent. ${ }^{11}$ Ipse enim Suetonius, etsi infamem omnibus vitiis eum proclamat, nihil certi ea de re nuntiare potest. ${ }^{12}$ Mulierum amator (de hoc mox infra), si etiam puerorum amore notus fuisset, num scholam publicam diu et prospere exercere potuisset? Sed nocet arrogans nimiumque superbus puerorum doctor, cui mediocres invident, meliores ad exemplum eius ipsi arrogantiores prodeunt. Ad hoc derisorem fuisse Remmium nullamque auctoritatis reverentiam habuisse satis manifestum est. ${ }^{13}$

De vanitate eius hactenus. Inter rumores, quos Suetonius congerere studuit, ut depravatos Palaemonis mores illustraret, unum reperit, qui ad libidines pertineret:

Sed maxime flagrabat libidinibus in mulieres usque ad infamiam oris: dicto quoque non infaceto notatum ferunt cuiusdam qui [quae?] eum in turba osculum sibi ingerentem quamquam refugiens devitare non posset, "Vis tu - inquit - magister, quotiens festinantem aliquem vides abligurrire?" (Gramm. 23, 6).

Quae corrupte tradita esse omnibus fere editoribus apparet. Duae igitur quaestiones solvendae sunt: prior de genere masculino, altera de ioci sensu. Cur mulierositatem Remmii fabella illustravit, cuius deuteragonistam virum esse masculini generis pronomina qui et aliquem clarissime monstrant? ${ }^{14}$ E magno emendationum numero notandae sunt tres: $<$ hominesque> post mulieres Ernout mares pro mulieribus Jernstedt et al. molles vel molliores Baldwin. Omnes videlicet mulieres aut excludere (ita Reifferscheid) aut viris adiungere aut in viros deformare aut saltem virili veste induere volunt. ${ }^{15}$

Verum tamen pronomen aliquis ad utrumque sexum generatim applicari potest. Vide etiam s. v. aliquis, ubi ad Propert. 2, 22, 5 expressis verbis dictum invenies formam masc. generis ad feminam pertinere. De accusativi sg. masc. aliquem usu v. etiam Apul. Met. 10, 2 in fine: cum videas aliquem sine corporis calore flagrantem. Sic de amantibus, sed praecipue de muliere cupidine plena. Cf. francogallicum quelqu'un. Nam aliqua admodum

11 Baldwin 1995, 381.

12 Tib. 45 et Galb. 22, quae affert Baldwin, nostra cum fabula male conferuntur. Nam de Remmii libidinibus arte et ambigue, quia argumenta prope nulla habuit, de Tiberii et Galbae aperte et copiose dixit.

13 „P's alleged arrogantia et luxuria would have been sufficient per se to render him suspect“: Kaster 2003, 235; cf. ibid. 231-232: „Elements in the sketch occasionally combine to produce an out-of-focus portrait: though notorious for vices that should have caused any right-minded parent to shun his school, P. was none the less the most successful gramm. of all surveyed in the DGR; though utterly sunk in self-indulgent luxury, he was none the less diligentissimus in the management of his res familiaris. The portrait as a whole is reminiscent of other stereotyped sketches of depraved and unworthy parvenus, including freedmen who were thought to have risen above their station."

14 Commate post libidinibus textus interpungitur a Kaster, op. cit. 26. Quod nec Latine nec iuste videtur. Cf. Baldwin 1995, 380: „I suppose the traditional text could be rescued by having (e.g.) commas after libidinibus and oris, thus creating separate statements of homosexual and heterosexual proclivity. But this is (to say the least) forced in itself, as well as leaving a peculiarly weak collocation of maxime... quoque and still being vulnerable to further objections."

15 Lectio mares a plurimis accepta est, sed sine feminarum notione rarissime mares invenies: Cic. Nat. deor. 2, 128: aliae mares aliae feminae sunt; Cels. 7, 26: ad mares tres, ad feminas duae; Apul. Apol. 38, 5: feminae subent, mares suriant et similia multa. Idem Suet. Claud. 33, 2: libidinis in feminas profusissimae marum omnino expers et Galba 22, ubi feminarum nota per ellipsin omissa est: libidinis in mares pronior [sc. quam in feminas]. His exemplis usus Baldwin lectionem mares approbatur. Perperam quidem. Et molliores quamquam probabilior reicienda tamen. Numquam enim absolute pro adulescentibus vel iuvenibus usurpatur. Etiam molles apud Petronium 23, 3 non absolute pace Baldwin, sed cum verbo spatalocinaedi coniunctum est adiectivo scilicet usu. Praeterea magistrum pueros tam perspicue in turba osculis adire temptasse quis credere potest? 
raro absolute usurpatur. Itaque ad aliquem prospiciens Medii Aevi librarius praecedens pronomen correlativum quae, quod in archetypo per unam litteram $q$ correptum vidit ${ }^{16}$, in masculinum qui vertit eo facilius, ut cuiusdam qui copulatio usitatissima esset, cuiusdam quae rarior oculis et manui appareret.

Nonnulli etiam part. festinantem corruptum censent ${ }^{17}$, quod tamen ceteri sensu translato pro coeunti vel masturbanti, i. e. quasi ad summum Veneris properanti, dictum esse lusumque verborum facetias dicto addere sentiunt. Sic ingeniosissime Housman eumque sequentes alii, qui osculum cum re obsceniore conferunt ${ }^{18}$. Sed (ab)ligurrire sensu obsceno nil aliud significat, quam mulieris pudenda lingere. Cuius usus exemplum dedit Suet. Tib. 45: hircum uetulum capreis naturam ligurrire ${ }^{19}$. Idem apud Ausonium invenies, Epigr. 87: Eunus Syriscus inguinum ligurritor etc; cf. eiusdem in eundem, 86: Eune, quod uxoris gravidae putria inguina lambis. Talia cum pueris 'festinantibus' parum congruunt. Itaque opprobrii amore cum Suetonio certare nolumus, sed de muliere eum scripsisse credimus. Et magister Palaemon in turba non a discipulo apellatus est, sed quia ludum Romae exercebat et doctrina notus erat (cf. ex. gr. Petr. 55, 5: rogo, inquit, magister, quid putas inter Ciceronem et Publilium interesse; Schol. ad Hor. Serm. 1, 9, 76 : sic Servius magister).

Restat pauca de ioco addere, cuius sal omnis in verbo abligurriendi positus est. Festinantem ad sua negotia puellam (libertinam fortasse talem, quam describit Hor. Carm. 1, 33, 14-16; Ep. 14, 15-16), senex attentus in turba videt ac simulata salutatione basio salaci subito oblinit. ${ }^{20}$ Quae hanc procacitatem tam lascive exprobravit, ut osculum Palaemonis cum voraciter edendo compararet. Verbo enim ligurriendi vel abligurriendi optimae Latinitatis auctores pro avide mandendo, comedendo, hauriendo, devorando et sim. saepissime utuntur (Ter. Eun. 936; Hor. Serm. 1, 3, 81; 2, 4, 79; Apul. Met. 10, 14; inde ligurritio vel abligurritio pro gula sagina voracitate arcessitur: Cic. Tusc. 4, 26; Script. Hist. Aug. 15, 2). Ergo abligurrire per hyperbolen ad immodice osculandum adhibuit hoc fere sensu: "Num inopinantem si quem vides, ilico tam avide osculas, ut comedere velle videaris?"21

${ }^{16}$ Simili vel eadem abbreviatura in MSS corripiuntur qui et quae: Capelli 1928, 301-302; Havet 1911, 182-183, \$\$ 775-776.

17 os tuentem coniecit Beck; fascinantem Christ, alia v. in app. crit. apud Brugnoli 1972, 26.

18 Housman 1931, 412: „Pro festinantem Reifferscheidius haesitantem, cuius dicti facetiae mihi non apparent. Quanto satius erat vel ab hoc uno loco discere festinare vulgari sermone dici qui in certamine venerio ad finem et seminis emissionem properat, iam cum praesagit gaudia corpus / atque in eost venus ut muliebria conserat arva (Lucr. 1106-1107)“. Eadem: Adams 1982, 144; Williams 2010, 398. Quorum vis non omnibus patebat. Baldwin (1995, 381-382: „Why should a person trying to escape the lust of Remmius Palaemon be himself on the point of orgasm?") nom. festinans proposuit sensusque nescioquos obsceno obsceniores Suetonio suo inculcare temptavit, „applying the seminal build up to Palaemon himself“. Quod iterum nec ad sensum nec ad rationes palaeographicas quadrat.

19 Fellationem nusquam significat. Contra Kaster 2003, 242 et Adams 1982, 140, sed errant. Et Catulli fr. 2: de meo ligurrire libido est false eodem ponunt. Hic enim ligurire idem est atque consumere (de Priapi horto): ita Nonius Marcellus, qui hunc versum affert (De compendiosa doctrina 195 Lindsay): ligurire, degustare, unde abligurire, multa avide consumere. Etiam in Cic. De domo sua 47, 15: quoniam iam dialecticus $<e s>$ et haec quoque liguris nihil obsceni; cf. Verr. 2, 3, 177; Tusc. 4, 26; Fam. 11, $21,5$.

${ }^{20}$ Non solum viros verum etiam mulieres basiando salutare Romani solebant: Mart. 10, 22; T. Rossi ap. Damschen, Heil, 2004, 108-10.

${ }^{21}$ Haud sane intellego, quomodo verba festinantem (mulierem scilicet!) abligurire etiam ad obsceniora alludere potuerint. Quibus tamen verbis Suetonius obscenum applicavit sensum vanitatis infamiae aliam immeritam adiungens. Nam infamiam oris ad lingendi stuprum attinere posse cum Kastner (2003, $240)$ et Baldwin $(1995,380)$ credimus. Quam propter ingrata oscula et praecipue propter abligurriendi verbum Suetonius Palaemoni intulit. 


\section{Opera citata}

Adams J. N. The Latin Sexual Vocabulary. London, Duckworth, 1982.

Baldwin B. The Sexual Tastes of Remmius Palaemon. Hermes 1995, 123, 380-382.

Barwick K. Remmius Palaemon und die römische ars grammatica. Leipzig, Dieterich'sche Verlagsbuchhandlung, 1922.

Brugnoli G. (ed.) C. Suetonii Tranquilli praeter Caesarum libros reliquiae. Pars prior. De grammaticis et rhetoribus. Leipzig, Teubner, ${ }^{2} 1972$.

Capelli A. Lexicon Abbreviaturarum. Leipzig, Weber ${ }^{2} 1928$.

Collart J. Palémon et l'ars grammatica. Revue de Philologie 1938, 64, 228-238.

Damschen G., Heil A. (Hgg.) Martial. Epigrammaton Liber Decimus. Das zehnte Epigrammbuch: Text, Uebersetzung, Interpretationen. Mit einer Einleitung, Martial-Bibliographie und einem rezeptionsgeschichtlichen Anhang. Frankfurt am Main, Lang, 2004.

Del Bello D. Forgotten Paths: Etymology and the Allegorical Mindset. Washington, The Catholic University of America Press, 2007.

Havet L. Manuel de critique verbale appliquée aux textes latins. Paris, Hachette 1911.

Housman A. E. Praefanda. Hermes 1931, 66, 402-412.

Kahn Ch. H. Pythagoras and the Pythagoreans: A Brief History. Indianapolis, Hackett Publishing Company, 2001.

Kaster E. A.(ed.) C. Suetonius Tranquillus. De grammaticis et rhetoribus. With transl., introd. and. comm. Oxford, Clarendon Press, ${ }^{2} 2003$.

Kolendo J.Éléments courants et exceptionnels de la carrière d'un affranchi. Le grammairien Q. Remmius Palaemon. Quaderni camerti di studi romanistici 1985, 13, 177-187.

Oliver R. P. The Claudian Letter I. AJA 1949, 53, 249-257.

Rawson E. Intellectual Life in the Late Roman Republic. Baltimore, The Johns Hopkins University Press, 1985. Sandys J.A History of Classical Scholarship from the Sixth Century B. C. to the End of the Middle Ages. Cambridge, CUP, 1903.

Williams C. A. Roman Homosexuality. Oxford, OUP, 22010.

For citation: Pozdnev M. M. De Remmii Palaemonis indole vitiisque. Philologia Classica 2016, 11(2), 253-257. DOI: 10.21638/11701/spbu20.2016.204

\section{О ХАРАКТЕРЕ И ПОРОКАХ РЕММИЯ ПАЛЕМОНА}

Михаил Михайлович Позднев

Рассказ Светония о Реммии Палемоне окрашен презрением к герою. Пороки Реммия отвечают его происхождению: биограф изображает прославленного грамматика тщеславным и недалеким выскочкой, к тому же сластолюбцем. По ходу повествования автор приводит некоторые, известные, вероятно, по школьным преданиям, высказывания Реммия. Слова с подозрительной точностью вписываются в портрет - хвастливы, однако и глупы до абсурда. Под конец Светоний вспоминает некую шутливую отповедь, полученную Реммием, по-видимому, в ответ на его вполне вольную манеру здороваться. Текст здесь неясен, убедительных исправлений не предложено, трактовки соперничают в обсценности. Выше пытаемся сперва восстановить контекст, затем оценить положение во-первых, вернуть словам Реммия остроумие, ожидаемое от человека, чье имя, став нарицательным, означало у римлян «грамматик» (по-нашему - «филолог»), и во-вторых, сообщить внятный, пусть в ущерб игривости, смысл последним строкам гл. 23 De grammaticis et rhetoribus. В итоге за грубыми штрихами Светония проступает образ, внушающий больше симпатии.

Received: 21.07 .2016

Final version received: 10.10 .2016 